\title{
THE INFLUENCE OF DIFFERENT TRAINING CONDITIONS ON THE KINEMATICS OF LONG JUMP-SPECIFIC EXERCISE IN YOUNG FEMALE JUMPERS
}

\author{
HUBERT MAKARUK ${ }^{1}$, ANDRZEJ MASTALERZ², MARCIN STARZAK ${ }^{1}$, \\ MARIUSZ BUSZTA ${ }^{3}$
}

\author{
Józef Piłsudski University of Physical Education in Warsaw, Faculty of Physical Education, \\ Department of Biomechanics ${ }^{2}$, Faculty of Physical Education and Sport in Biała Podlaska, \\ Department of Athletics ${ }^{1}$, Department of Foreign Languages ${ }^{3}$
}

\author{
Mailing address: Hubert Makaruk, Faculty of Physical Education and Sport in Biała Podlaska, \\ Department of Athletics, 2 Akademicka Street, 21-500 Biała Podlaska, \\ tel.: +48 83 3428779, fax: +48 83 3428700, e-mail: hubert.makaruk@awf-bp.edu.pl
}

\begin{abstract}
Introduction. This study examined the changes in the kinematic parameters of long jump-specific technical exercise performed in different training conditions. Material and methods. The study involved a group of young female athletes who volunteered to participate in the research. The key variables for long jump performance were measured using the Xsens MVN system. A three-way ANOVA (general linear model with repeated measures; factors: surface $\mathrm{x}$ hurdle $\mathrm{x}$ number of jumps) was used to determine if significant differences existed between the testing conditions. Results. The main finding of this study was that the tartan surface resulted in significantly $(p<0.05)$ greater velocities of the centre of mass of the body $(\mathrm{CM})$ or parts of the athlete's body than the grass surface. The second important finding was that the hurdles condition provided significantly $(p<0.05)$ greater velocity of the CM when landing and shorter contact time compared to the condition without hurdles. Conclusions. The findings of the study indicate that technical exercise should be performed on harder surfaces such as a tartan track rather than softer ones (e.g. grass) due to more beneficial movement characteristics and greater potential for the automaticity of movement during specific training tasks.
\end{abstract}

Key words: take-off, touch-down, athletics jumps, technical skills

\section{Introduction}

The long jump is incorporated into technical athletics events due to the complexity of the movement pattern. This highlights the leading role of technical skills among the factor which assure superior performance in athletics and the importance of technical issues in the entire process of training. Good technique is associated with better performance, although better performance does not indicate better technique. This statement shows that technique should not be described by the same variables as performance or the result of performance [1]. For example, the approach speed determines performance in the long jump, but the velocity of the free leg at take-off describes the long jump technique.

The technique of the long jump is most often characterised using kinematic parameters, that is through quantitative analysis. Long jump performance has been described by researchers very often and in varying detail, supporting coaches in recognising key variables in each phase of the long jump. For example, it is known that the distance of the long jump is strongly determined by the athlete's horizontal velocity at the end of the approach phase [2,3] and that the relationship between jump distance and horizontal approach velocity is not linear for an individual jumper [4]. It is also known that top long jumpers maintain their normal running action until about 2-3 strides before take-off [5]. The knowledge that is being acquired about the long jump is becoming increasingly specialised. Lees et al. [1] report that early research focused mainly on the approach and take-off phases. Later researchers $[3,6]$ started to analyse the whole phase from touch-down to take-off in detail, and it turned out this more comprehensive analysis is critical for determining whether take-off is appropriate. However, all of these parameters describe long jumps from a full-length approach in the standard condition, while technical exercises are mainly performed from a shortened approach and in a non-complex form during training [7].

In one of the few studies that directly examined technical exercises, Bober [8] found that the velocity at take-off and movement time of the lower extremities at the last stride in the shortened approach in the long jump are significantly lower than those in the full approach. Other researchers have evaluated the effects of different conditions on long jump technique. In one of the studies [9] the athletes executed long jumps with a medium length approach in standard conditions, on upward inclined boards, and on raised flat boards. The inclined board increased the vertical velocity of the centre of mass of the body $(\mathrm{CM})$ at take-off and provided more time in the flight phase, whereas the raised flat board resulted in a greater pivot of the body over the take-off foot. Both the raised flat board and the inclined board changed the technique of the jump. The above mentioned cases show that long jump training should be designed based on systematic analyses of the technique of exer- 
cises that are targeted at the development of technical skills. Without such research and the dissemination of its results, the work of coaches is based only on their intuition.

If an athlete is to develop a high level of sports technique, methods and exercises that are strongly linked with the demands of a given sport need to be used. In order to ensure a high specificity of training in the long jump, many technical exercises are performed on a tartan surface. This allows athletes to increase the velocity of movement and enhances the transfer of training gains to the competitive condition. On the other hand, however, high velocities of movement result in large impact forces and may lead to overloading the movement apparatus [10]. The potential for injury is reduced if the athlete performs exercises on natural, more compliant surfaces (e.g. grass). The other common practice in long jump technical training is employing sports equipment. Very often coaches use hurdles, distance indicators in the sand pit, or a hanging ball to improve some detail of the athlete's movement technique [7].

To our knowledge, no study has so far examined the differences between exercise that imitates the take-off phase in the long jump in different surface conditions and with and without the use of hurdles. That is why the aim of this study was to determine if different ground surfaces and using hurdles during long jump technical exercise influence kinematics in the take-off and landing phases. It was hypothesised that there are significant differences between kinematic parameters in the training conditions which were compared. We expected that the tartan surface would produce characteristics that are closer to the competitive conditions for performing the long jump compared to the grass surface.

\section{Material and methods}

\section{Subjects}

Six young female athletes volunteered to participate in the study (mean age $=15.8$ years, $S D=0.7$; body height $=1.63$ $\mathrm{m}, \mathrm{SD}=0.4$; body mass $=59 \mathrm{~kg}, \mathrm{SD}=4$ ). They were experienced in technical long jump exercises and injury-free. The study was approved by the Senate Research Ethics Committee of the University of Physical Education in Warsaw. Before the study started, the athletes and their parents gave their written consent.

\section{Testing procedures}

The athletes were asked to do a special take-off exercise that involved 5 take-offs with 3 strides between them performed with a-5-stride approach (fig. 1). They performed this exercise in all four conditions (with hurdles on grass, with hurdles on tartan, without hurdles on grass, and without hurdles on tartan) in a within-subject design. The order of conditions was counterbalanced across the subjects. Three trials were done for each condition with 3-minute rest to avoid fatigue. The data were collected during the athletes' preparatory season (in April). The warm-up consisted of a 10-minute jog, 8-minute dynamic stretching, 4 × $20 \mathrm{~m}$ of skipping, and $2 \times 20$ $\mathrm{m}$ of unilateral rebounds. Before the tests were conducted, the subjects were told that their goal was to perform this exercise as best as possible. The exercise was performed on a track surface (tartan) and on a stadium field (grass) in running shoes. The hurdles were individually adjusted and spaced 9.40-9.70 $\mathrm{m}$ apart in order for the athletes to execute three steps between the hurdles. The height of hurdles was $0.4 \mathrm{~m}$.

The kinematic parameters analysed in this study were evaluated using the Xsens MVN system (Xsens Technologies
B.V., Ensched, Holland) [11]. This full-body inertial kinematic system consists of 17 Motion Tracker sensors (Mtxs) and 2 Xbus Master units (XMs) that are built into the MVN Lycra suit system. The Mtxs include 3D rate gyroscopes, 3D linear accelerometers, and 3D magnetometers. The XMs interconnect the MTx sensors, deliver power to the sensors, and collect the data. The system provided a wireless capture of the 3 degrees of body movement with a sampling frequency of $120 \mathrm{~Hz}$. Before each trial, the basic N-pose calibration procedure was performed. The MVN Studio (version 3.3.0) graphical software was used to record and analyse the captured data. The following parameters were measured in the take-off phase: the velocity of the body's centre of mass (CM) at touch-down was taken during the first contact between foot and ground, the velocity of the $\mathrm{CM}$ at take-off was taken during the last contact between foot and ground, the maximum velocity of the foot during the last stride was taken to be the highest value of velocity during the last stride, and contact time of the take-off foot was determined by measuring the time period from foot touch-down to take-off. One parameter was measured during the landing phase: it was the velocity of the CM at touch-down after the jump which was taken at the first contact between foot and ground after the jump.

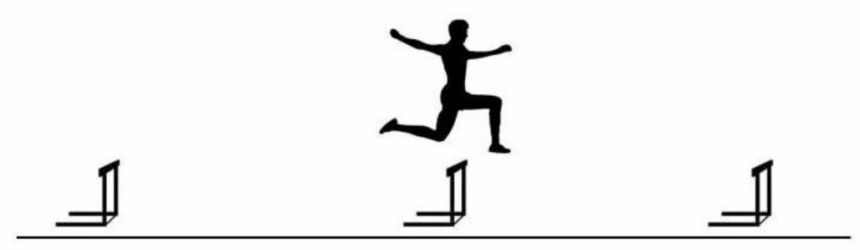

Figure 1. Illustration of jumps with hurdles

All of the 5 take-offs in the 12 trials performed by each athlete were considered in the analysis. The reliability of the measurements was assessed by calculating intraclass correlation coefficients (ICC), and the ICCs were high (0.87-0.95).

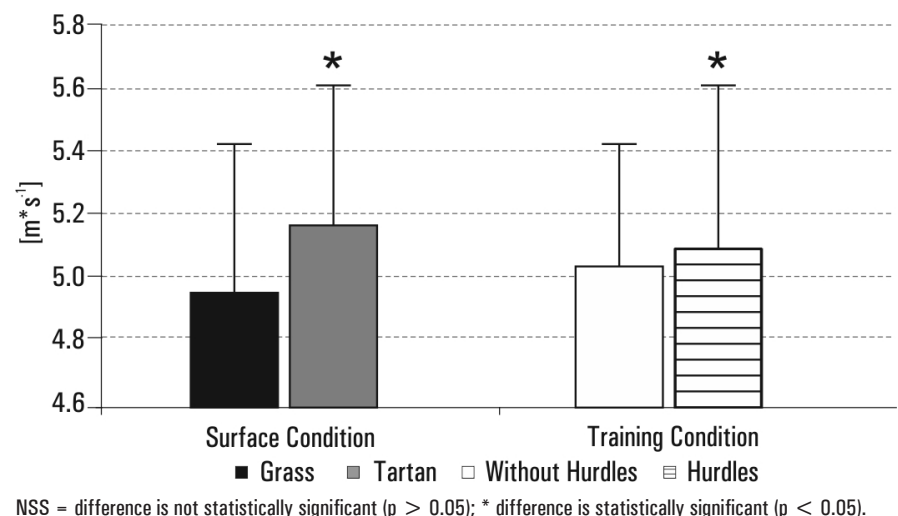

Figure 2. Mean (SD) velocity of CM at touch-down for different surface and training conditions

\section{Data analysis}

All the tested parameters were normally distributed, and a three-way ANOVA (general linear model with repeated measures; factors: surface $\mathrm{x}$ hurdle $\mathrm{x}$ number of jumps) was used to determine if significant differences existed between testing 
conditions. Significant main effects were further analysed with a Tukey-adjusted pairwise comparison of within-subject differences for the parameters. Significance was set at a level of $\mathrm{p}<$ 0.05. Statistica for Windows version 10 (Poland) software was used for all statistical calculations.

\section{Results}

\section{Velocity of CM at touch-down}

The results obtained for this parameter are presented in figures 2-3. It was revealed that there was a main effect of surface $\left(\mathrm{F}_{1.5}=16.33 ; \mathrm{p}<0.01\right)$ and jumps $\left(\mathrm{F}_{4,20}=10.75 ; \mathrm{p}<0.001\right)$ on this parameter. A post-hoc analysis showed that the tartan surface produced greater velocity than the grass surface. It was also observed that the velocity at the first take-off was lower than in the consecutive jumps.

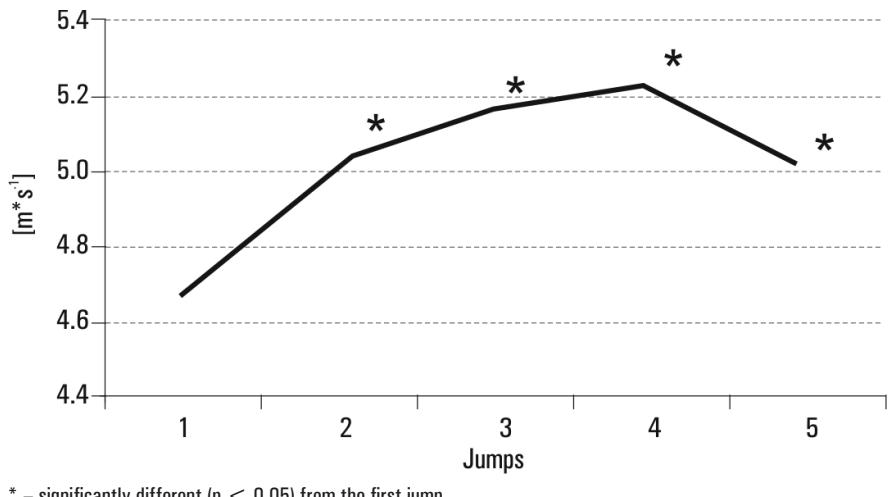

* - significantly different $(\mathrm{p}<0.05)$ from the first jump.

Figure 3. Mean (SD) velocity of CM at touch-down during consecutive jumps

\section{Velocity of CM at take-off}

The results obtained for this parameter are shown in figures 4-5. The main effects of surface $\left(\mathrm{F}_{1.5}=23.17\right.$; $\left.<<0.01\right)$ and jumps $\left(\mathrm{F}_{420}=18.46 ; \mathrm{p}<0.001\right)$ were found. The results of this analysis revealed that the tartan surface provided greater velocity than the grass surface and demonstrated that velocity at the first jump was lower than in the case of the second, third, and fourth jumps.

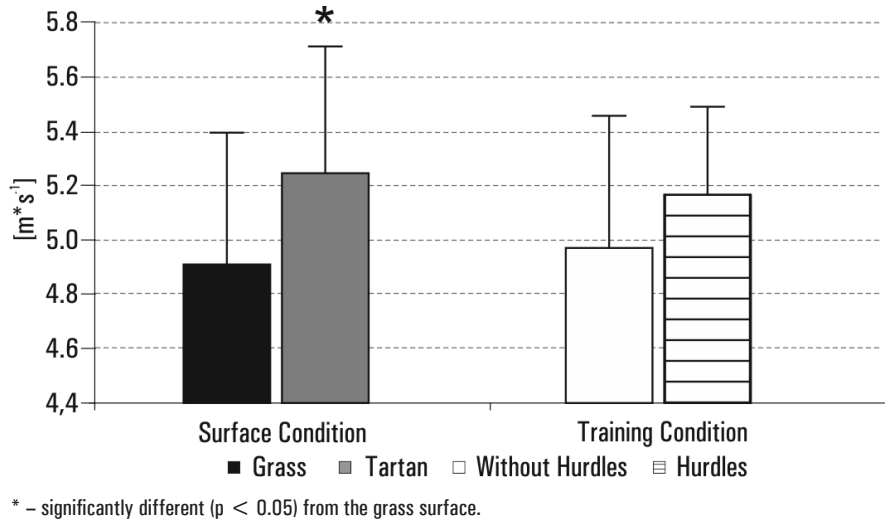

Figure 4. Mean (SD) velocity of CM at take-off for different surface and training conditions

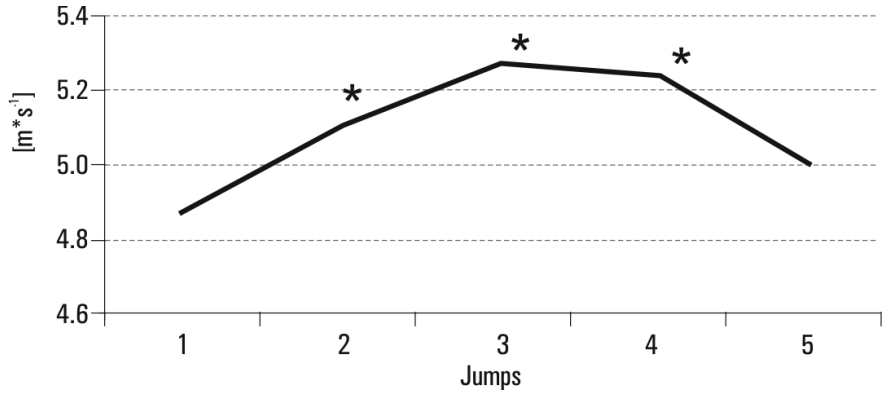

Figure 5. Mean (SD) velocity of CM at take-off during consecutive jumps

\section{jump) \\ Velocity of CM at touch-down during landing (after the}

The results obtained for this parameter are illustrated in figures 6-7. The main effects of hurdles $\left(\mathrm{F}_{1.5}=7.31 ; \mathrm{p}<\right.$ $0.05)$, surface $\left(\mathrm{F}_{1.5}=6.75 ; \mathrm{p}<0.05\right)$, and jumps $\left(\mathrm{F}_{420}=11.27\right.$; $\mathrm{p}<0.001$ ) were revealed. Using hurdles resulted in greater velocity than performing the task without hurdles, and the tartan surface provided greater velocity compared to the grass surface. The velocity of the last jump was lower than in the preceding jumps.

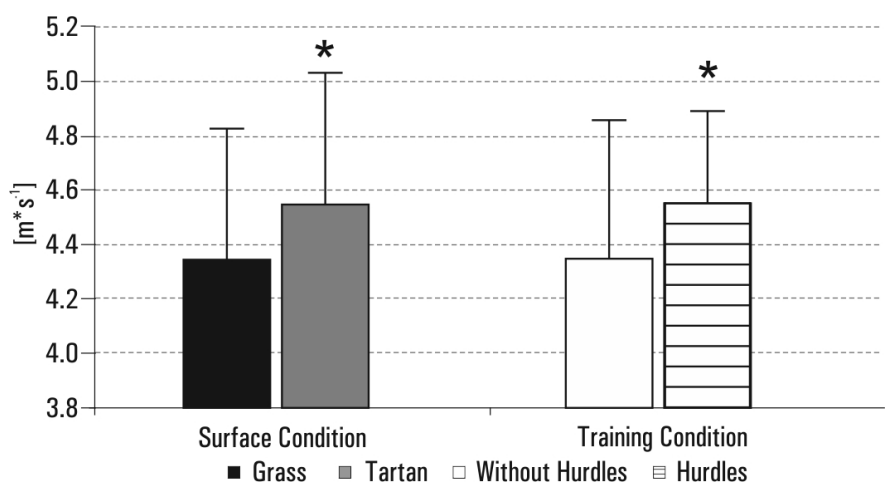

* - significantly different $(\mathrm{p}<0.05)$ from the grass surface; significantly different $(\mathrm{p}<0.05)$ from the conditions without hurdles.

Figure 6. Mean (SD) velocity of CM at touch-down during landing for different surface and training conditions

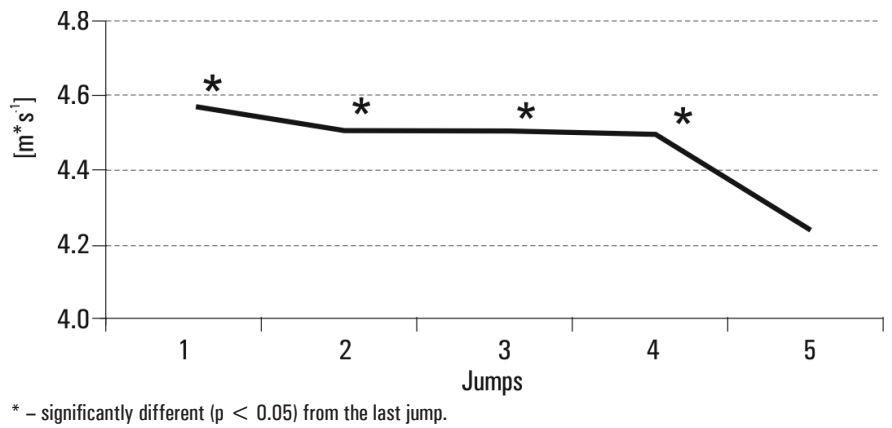

Figure 7. Mean (SD) velocity of CM at touch-down during landing for consecutive jumps 


\section{Maximum velocity of foot during last stride}

The results obtained for this parameter are presented in figures 8-9. There were significant main effects of surface $\left(\mathrm{F}_{1.5}=9.04 ; \mathrm{p}<0.01\right)$ and jumps $\left(\mathrm{F}_{4.20}=13.63 ; \mathrm{p}<0.001\right)$. The tartan surface provided higher velocity compared to the grass surface. It was found that the velocity at the first jump was lower than that in the consecutive jumps.

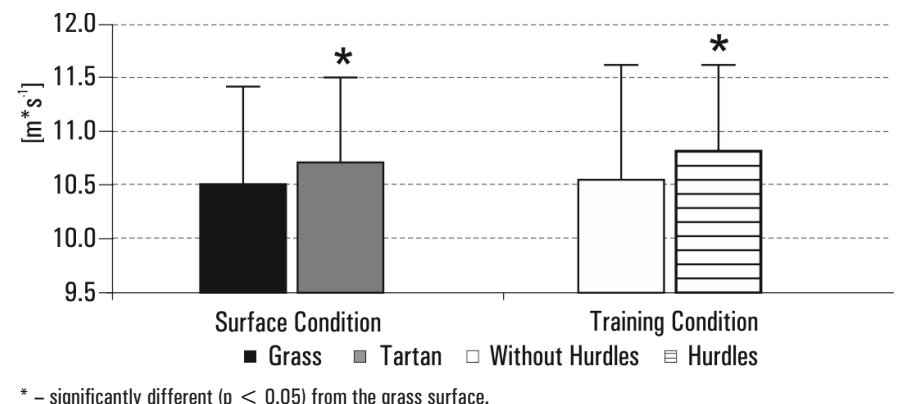

Figure 8. Mean (SD) velocity of foot during last stride before take-off for different surface and training conditions

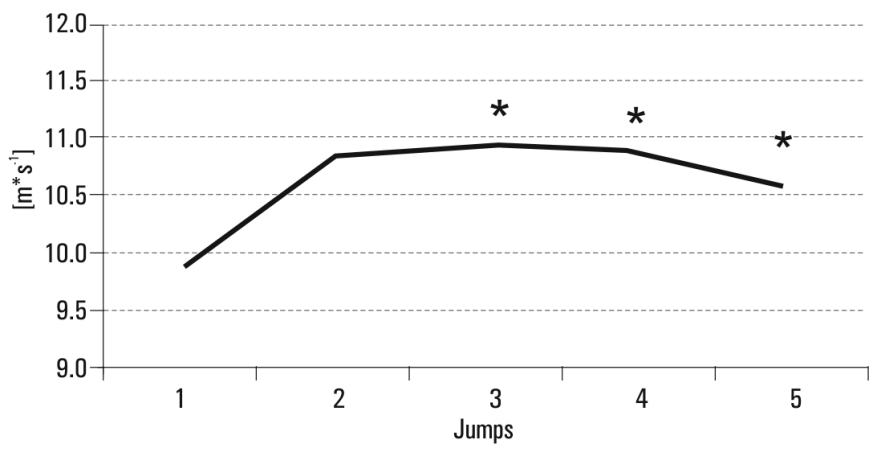

${ }^{*}$ - significantly different $(p<0.05)$ from the first jump.

Figure 9. Mean (SD) velocity of foot during last stride before take-off for consecutive jumps

\section{Contact time of take-off foot}

The results obtained for this parameter are presented in figures 10-11. The main effect of hurdles $\left(\mathrm{F}_{1.5}=10.18 ; \mathrm{p}<0.05\right)$ in this parameter was revealed. The hurdle jumps had a shorter contact time than the jumps without hurdles.

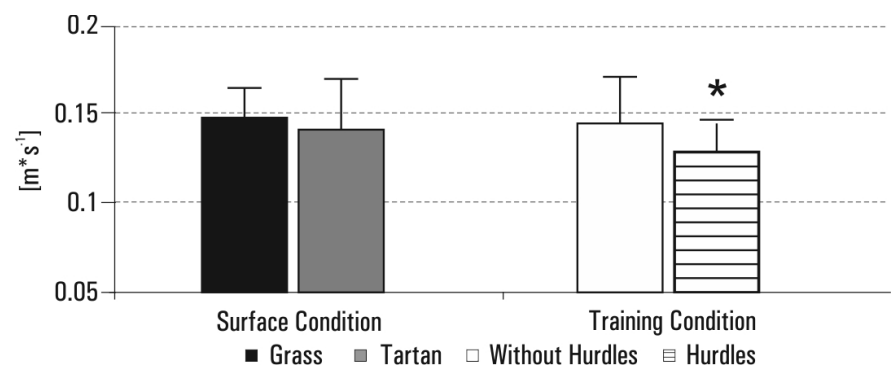

${ }^{*}$ - significantly different $(p<0.05)$ from the conditions without hurdles.

Figure 10. Mean (SD) contact time for different surface and training conditions

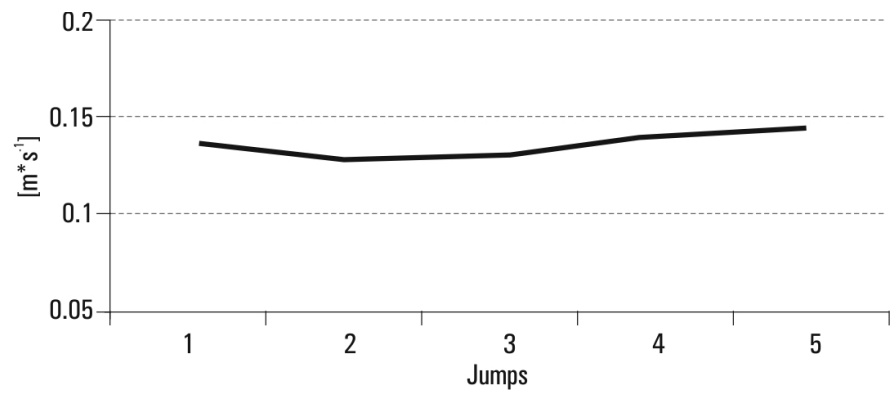

Figure 11. Mean (SD) contact time for consecutive jumps

\section{Discussion}

The crucial role of horizontal velocity is well established in a deterministic model of the long jump $[2,3,4,5,6]$. The following variables are most often used to describe this model: speed at take-off, horizontal velocity at take-off, and horizontal velocity in the fourth and last strides. However, Lees [1] and Graham-Smith and Lees [12] identified several limitations of this approach. In their opinion, the importance of technique was ignored in this model. Previous authors [8, 9] also reported that there is a lack of studies which imitate real jumping movement in long jump performance. Therefore, the aim of this study was to determine if different ground surfaces and using hurdles during long jump technical exercise influence kinematics in the take-off and landing phases.

This study can be useful because it provides information about changes in technique in the most common technical exercises in the long jump due to different training conditions. As stated in the definition of sports technique [13], our analysis included "a specific sequence of movements or parts of movement in solving movement tasks in sports situations". The values and relations between the velocity and time parameters of the sub-phases of the take-off and landing phases were the primary criteria of assessing the effectiveness of the athletes' technique in this study. As was expected, we found that the type of training surface and sports equipment (hurdles) influenced the technique of a specific sports task in the long jump. The main finding of this study was that the tartan surface resulted in greater velocities of the CM or parts of the athletes' bodies. The second important finding was that the hurdle condition provided a greater velocity of the CM during landing (after the jump) and shorter contact time compared to the condition without hurdles.

It was found already in early research that touch-down velocity has a substantial effect on jumping distance, even when approach speeds are similar [14]. The important role of takeoff velocity for the performance and technique of a long jump was also previously observed [12]. Research by Miladinov [15], for instance, showed that fast movement of the take-off leg towards the board during the last step put the athlete's body in a position which was favourable to performing an effective take-off. This means that the variables of velocity in different phases of the long jump may describe the technique of this sports exercise.

Based on previous studies [16, 17], we assumed that the velocity of the CM at touch-down and take-off, maximum velocity of the take-off foot, and velocity of the CM at touch-down during landing would be greater on the tartan surface compared with the grass surface. Our results suggest that the tartan surface produces characteristics that are closer to the competitive conditions in the long jump. This is a major reason why a hard synthetic surface may provide better outcomes in technical training. A possible mechanism for these changes may be 
related to the ability of the surface to absorb the impact ground reaction force (GRF). Harder synthetic surfaces such as tartan absorb a smaller percentage of the GRF generated upon impact than softer surfaces (e.g. grass). Therefore, synthetic surfaces produce greater GRF than a grass surface during athletic activities [17]. GRF is an important factor for jumping and running activities, because it was found that improved generation of muscular force and its transmission to the ground play a key role in increasing movement speed at take-off and running [18].

Although CM velocity changed at touch-down and take-off depending on the surface, the contact time was similar for both surface conditions. The findings regarding the relationship between contact time and horizontal velocities in the literature are inconsistent. Beres et al. [19], who used different lengths of approach which included 6, 8, 10, and 12 strides, found significant changes in velocity at take-off and touch-down but none in contact time during the take-off phase. In contrast, Nemtsev et al. [20] showed take-off velocity significantly increased and contact time significantly decreased with 3,8 , and 12 strides of approach. It is important to highlight that contact time in the take-off phase did not correlate with the jumping distance [15]. This result may support the assumption tested in previous research that the contact time of the take-off phase should be optimal and that it depends on individual factors. Attempts to find a training solution that would decrease contact time are needed because young female athletes have a tendency to increase contact time during the take-off phase [7]. It must be noted that shorter contact time with the ground results in more effective elastic energy for powerful movements by utilising the stretch-shortening cycle [21, 22].

Of major interest to us was observing if employing sports equipment would influence the technique of jumping exercise. It was satisfying to see that contact time decreased and the velocity of the CM at touch-down during landing increased when hurdles were incorporated into the performance of a jumping task. In addition, there was also a trend ( $p=0.07)$ towards higher velocity at take-off in hurdle conditions. Hurdles were used in technical training in the long jump in this study as extrinsic motivators, since other studies have found that using sports equipment, such as a suspended ball in the vertical jump [23] or a cone in the standing long jump [24], improves jumping performance. The current findings are in agreement with the predictions of the constrained action hypothesis that explains the motor learning and performance benefits observed when athletes adopt an external relative to an internal focus of attention. This hypothesis suggests that directing non-conscious (external) attention towards the movement of an athlete's body during motor skill performance facilitates automatic cognitive processing. This assistance allows the motor control system to self-organise more naturally, without overloading the central and peripheral nervous systems [25], and thus results in fast and accurate movements. In contrast, when attention is directed internally, towards the movement of an athlete's body or part of the body, automatic processing is disturbed. This interruption of the motor control system hampers motor skills. Despite these convincing findings, it is interesting that a study by Porter et al. [26] involving elite track-and-field athletes showed they usually received feedback that promoted an internal focus of attention. Based on the above-mentioned findings and the current findings, it is logical to suggest that exercises involving sports equipment should be incorporated into technical training programmes for the long jump.

Further observations showed that all the analysed velocities were characteristically different across the five consecutive take-offs. The characteristics describing the first jump produced lower velocities than those in the following jumps. This fact may be caused by the early acceleration phase for the first jump compared to the following jumps. Therefore, it would be recommended to employ more than one hurdle if the training is to develop technical skills. We also observed a significant decrease of velocity during the landing phase of the last jump. We would speculate that the athletes did not execute an active landing of the foot due to the fact that they stopped the exercises prematurely or due to fatigue. This issue was addressed by our previous study in plyometric training [27].

The current study has several limitations, which at the same time offer directions for future research. First of all, this study utilised a small sample. Therefore, replication in larger sample is needed. The second limitation of this research is that we examined only young female athletes. Future studies should be conducted on adult male and female athletes to see if similar motor behaviours are replicated. It would also be interesting to use other surfaces (e.g. paths) that are much harder than the grass surface but absorb ground reaction forces better than the tartan surface.

\section{Conclusions}

Training on the grass enables young jumpers to learn a basic take-off technique in the long jump. Additionally, it makes it possible to implement greater volumes of training as this surface absorbs ground reaction forces during landing. However, our study indicates that technical exercises should be performed on a harder surface, such as tartan, rather than a softer one (e.g. grass) due to more beneficial movement characteristics, including the greater velocities of the CM at touchdown and take-off. We have also found that the hurdle condition provided a greater velocity of the CM during landing (after the jump) and shorter contact time compared to the condition without hurdles. This may be seen as evidence proving greater automaticity of movement during specific training tasks.

\section{Acknowledgements}

The research was financed from grant number RSA1 002251 for the "Development of Sports at University" awarded by the Polish Ministry of Science and Higher Education.

\section{Literature}

1. Lees A. (2002). Technique analysis in sports: a critical review. Journal of Sports Sciences 20(10), 813-828.

2. Hay J.G., Miller J.A., Canterna R.W. (1986). The techniques of elite male long jumpers. Journal of Biomechanics 19(10), 855-866.

3. Lees A., Graham-Smith P., Fowler N. (1994). A biomechanical analysis of the last stride, touchdown, and takeoff characteristics of the men's long jump. Journal of Applied Biomechanics 10, 61-61.

4. Bridgett L.A., Linthorne N.P. (2006). Changes in long jump take-off technique with increasing run-up speed. Journal of Sports Sciences 24(8), 889-897.

5. Hay J.G., Nohara H. (1990). Techniques used by elite long jumpers in preparation for takeoff. Journal of Biomechanics 23(3), 229-239.

6. Lees A., Fowler N., Derby D. (1993). A biomechanical analysis of the last stride, touch-down and take-off characteristics of the women's long jump. Journal of Sports Sciences 11(4), 303-314.

7. Makaruk H., Sadowski J. (2008). Selected aspects of long and triple jump training. Biała Podlaska: ZWWF. [in Polish]

8. Bober T. (1973). Biomechanical principles of technical training. Wrocław: AWF. [in Polish]

9. Koyama H., Muraki Y., Ae M. (2005). Athletics: Effects of 
an inclined board as a training tool on the take-off motion of the long jump. Sports Biomechanics 4(2), 113-129.

10. Boden B.P., Dean G.S., Feagin Jr J.A., Garrett Jr W.E. (2000). Mechanisms of anterior cruciate ligament injury. Orthopedics 23(6), 573-578.

11. Roetenberg D., Luinge H., Slycke P. (2013). Xsens MVN: Full 6DOF Human Motion Tracking Using Miniature Inertial Sensors. Xsens Technologies, Enschede, The Netherlands.

12. Graham-Smith P., Lees A. (2005). A three-dimensional kinematic analysis of the long jump take-off. Journal of Sports Sciences 23(9), 891-903.

13. Beyer E. (1992). Dictionary of sport science. Schorndorf: Verlag Karl Hofmann.

14. Seyfarth A., Friedrichs A., Wank V., Blickhan R. (1999). Dynamics of the long jump. Journal of Biomechanics 32(12), 1259-1267.

15. Miladinov O. (2006). New aspects in perfecting the long jump technique. New Studies in Athletics 21(4), 7-25.

16. Brosnan J.T., McNitt A.S., Serensits T.J. (2009). Effects of varying surface characteristics on the hardness and traction of baseball field playing surfaces. International Turfgrass Society Research Journal 11, 1053-1065.

17. Nigg B.M., Yeadon M.R. (1987). Biomechanical aspects of playing surfaces. Journal of Sports Sciences 5(2), 117-145.

18. Weyand P.G., Sternlight D.B., Bellizzi M.J., Wright S. (2000). Faster top running speeds are achieved with greater ground forces not more rapid leg movements. Journal of Applied Physiology 89(5), 1991-1999.

19. Béres S., Csende Z., Lees A., Tihanyi J. (2014). Prediction of jumping distance using a short approach model. Kinesi- ology 46(1), 88-96.

20. Nemtsev O., Doronin A., Nemtseva N., Sukhanov S., Shubin M. (2014). Features of takeoff phase in long jumps with various run-up lengths. In 32nd Conference of the International Society of Biomechanics in Sports, July 12-16, 2014, Johnson City, USA: East Tennessee State University.

21. Hay J.G. (1999). Changes in muscle-tendon length during the take-off of a running long jump. Journal of Sports Sciences 17(2), 159-172.

22. Kyröläinen H., Avela J., Komi P.V. (1989). Regulation of muscle force and stiffness during long jump take off. Journal of Biomechanics 22(10), 1042.

23. Ford K.R., Myer G.D., Smith R.L., Byrnes R.N., Dopirak S.E., Hewett T.E. (2005). Use of an overhead goal alters vertical jump performance and biomechanics. The Journal of Strength \& Conditioning Research 19(2), 394-399.

24. Porter J.M., Anton P.M., Wu W.F. (2012). Increasing the distance of an external focus of attention enhances standing long jump performance. The Journal of Strength \& Conditioning Research 26(9), 2389-2393.

25. Latash M.L., Turvey M.T., Bernstein N.A. (1996). Dexterity and its development. Lawrence Erlbaum.

26. Porter J., Wu W., Partridge J. (2010). Focus of attention and verbal instructions: strategies of elite track and field coaches and athletes. Sport Science Review 19(3-4), 77-89.

27. Makaruk H., Czaplicki A., Sacewicz T., Sadowski J. (2014). The effects of single versus repeated plyometrics on landing biomechanics and jumping performance in men. Biology of Sport 31(1), 9-14.

Submitted: February 12, 2015

Accepted: September 2, 2015 